\title{
Education and Training Needs in the Radiation Sciences: Problems and Potential Solutions
}

Author(s): Joseph R. Dynlacht, Elaine M. Zeman, Kathryn D. Held, James Deye, Bhadrasain Vikram, and Michael C. Joiner

Source: Radiation Research, 184(5):449-455.

Published By: Radiation Research Society

DOI: http://dx.doi.org/10.1667/RR14199.1

URL: http://www.bioone.org/doi/full/10.1667/RR14199.1

BioOne (www.bioone.org) is a nonprofit, online aggregation of core research in the biological, ecological, and environmental sciences. BioOne provides a sustainable online platform for over 170 journals and books published by nonprofit societies, associations, museums, institutions, and presses.

Your use of this PDF, the BioOne Web site, and all posted and associated content indicates your acceptance of BioOne's Terms of Use, available at www.bioone.org/page/terms_of_use.

Usage of BioOne content is strictly limited to personal, educational, and non-commercial use. Commercial inquiries or rights and permissions requests should be directed to the individual publisher as copyright holder. 


\title{
Education and Training Needs in the Radiation Sciences: Problems and Potential Solutions
}

\author{
Joseph R. Dynlacht, ${ }^{a, 1,2}$ Elaine M. Zeman, ${ }^{b, 2}$ Kathryn D. Held, ${ }^{c, 2}$ James Deye, ${ }^{d, 2,3}$ Bhadrasain Vikram ${ }^{e, 2}$ and \\ Michael C. Joiner ${ }^{f, 2}$ \\ ${ }^{a}$ Department of Radiation Oncology, Indiana University School of Medicine, Indianapolis, Indiana 46202-5116; ${ }^{b}$ Department of Radiation \\ Oncology, University of North Carolina School of Medicine, Chapel Hill, North Carolina 27599-7512; ' Department of Radiation Oncology, \\ Massachusetts General Hospital and Harvard Medical School, Boston, Massachusetts 02114-2621; ' National Cancer Institute, Division of Cancer \\ Treatment and Diagnosis, Radiation Research Program, Bethesda, Maryland 20892-9727; ' Clinical Radiation Oncology Branch, NCI/NIH, \\ Bethesda, Maryland 20892-9727; and ${ }^{f}$ Department of Oncology, Wayne State University School of Medicine, Detroit, Michigan 48201-2013
}

Dynlacht, J. R., Zeman, E. M., Held, K. D., Deye, J., Vikram, B. and Joiner, M. C. Education and Training Needs in the Radiation Sciences: Problems and Potential Solutions. Radiat. Res. 184, 449-455 (2015).

This article provides a summary of presentations focused on critical education and training issues in radiation oncology, radiobiology and medical physics from a workshop conducted as part of the 60th Annual Meeting of the Radiation Research Society held in Las Vegas, NV (September 21-24, 2014). Also included in this synopsis are pertinent comments and concerns raised by audience members, as well as recommendations for addressing ongoing and future challenges. ๑ 2015 by Radiation Research Society

\section{INTRODUCTION}

At the 60th Annual Meeting of the Radiation Research Society (RRS) held in Las Vegas, NV (September 21-24, 2014), a workshop was held entitled "Education and Training Needs in the Radiation Sciences: Problems and Potential Solutions." Speakers and audience members were encouraged to identify problems (ongoing and more recently realized) in the education and training of the next generation of radiation scientists and discuss how these issues might be addressed. The discussions emphasized radiation oncology-related issues, reflecting the current areas of interest of many RRS members. This article provides a synopsis of the workshop and some proposed recommendations applicable to students and early career scientists who are actively seeking training and mentorship in the radiation sciences, as well as those tasked with

\footnotetext{
${ }^{1}$ Address for correspondence: Indiana University, Department of Radiation Oncology, Indiana Cancer Pavilion, RT041, 535 Barnhill Dr., Indianapolis, IN 46202; e-mail: jdynlach@iupui.edu.

2 All authors contributed equally to this article.

3 Retired.
}

teaching radiation biology courses and/or serving as research mentors. Given the nature of the workshop and this article, some of the content herein represents personal opinions and conjecture based on the perspectives of the participants.

\section{DECLINING WORKFORCE IN RADIATION ONCOLOGY-RELATED EDUCATION AND RESEARCH}

In the last decade, several published articles $(1-4)$ have reported on a continuing decrease in the number of individuals having sufficient formal and informal training in the radiation sciences to provide education and training, as well as guide research programs, in radiation biology and physics, particularly with regard to radiation oncology and radiation protection. Coupled with this are declines in the membership of some radiation-related professional societies. For example, the RRS reports that the number of full members declined by more than $20 \%$ between 2003 and 2012, and the Health Physics Society (HPS) has seen a $35 \%$ decline in plenary membership between 2001 and 2013 (5). If this trend continues, it will negatively impact future generations of scientists and physician-scientists who are charged with shaping the future of radiation oncology, medical physics and the radiation sciences, in general. Several reasons for the decline have been identified $(1-4)$, but perhaps the primary one is that most trainees pursuing academic careers in oncology, who will be responsible for mentoring the next generation of radiation researchers and physician-scientists, do not self-identify as radiation biologists, have never taken a formal course in radiobiology and have only a rudimentary understanding of basic radiation physics and dosimetry.

There is concern that biology researchers with limited formal training in, or even exposure to, radiation biology and physics are less likely to know and/or seek out medical physicists and radiation oncologists to collaborate with. Yet 
those latter groups have increasing interest in collaborating on radiation biology-related translational research. Furthermore, given the ever-increasing complexity of radiationgenerating devices in today's laboratories and clinics, a lack of training in basic principles of radiation dosimetry and measurement has grave implications for research. Since translational and clinical research increasingly requires teams of investigators with differing expertise, such as physicists, biologists, physicians and statisticians, it is critical that all team members be able to communicate well. Hence, a general understanding of both the biological and physical basis of contemporary radiation therapy is necessary. Training to develop this skill set is crucial for producing individuals who can serve as future experts and mentors. Yet, a running theme through the workshop discussions was the vicious cycle of a decreasing radiation sciences workforce leading to fewer qualified educators/ mentors, which results in fewer students entering the radiation sciences, which in turn, further exacerbates the workforce situation.

\section{RADIATION/CANCER BIOLOGY EXAMS FOR RADIATION ONCOLOGY: OUTLINES AND PRACTICE EXAMS, BUT NO DETAILED CURRICULUM}

Two of the major constituencies requiring radiation biology education are the medical and physics residentsin-training in radiation oncology and radiology, yet, not only is there a decreasing number of trained radiobiologists to provide the education (1-4), but, even with excellent textbooks on radiation biology for the radiation oncology community, there is a paucity of other educational materials to aid instructors and students. The American Board of Radiology (ABR) creates and administers board certification exams for radiation oncology and medical physics and maintains outlines of biology and physics topics that need to be taught for the exams. As part of the board certification process for radiation oncologists, candidates are required to pass a written exam in radiation and cancer biology, written exams in radiation physics and clinical radiation oncology and an oral clinical exam. The biology questions cover the gamut of radiation oncologyrelevant topics, from basic interactions of radiation with matter, through molecular and cellular damage/repair and cell death processes, modifiers of radiation response, normal tissue radiobiology, tumor biology, biological underpinnings of modern dose fractionation techniques in the clinic, radiation carcinogenesis and radiation protection.

In 2014, the ABR updated its outline of radiation and cancer biology topics covered on the radiation biology certification exam (available at http://www.theabr.org/ ic-ro-study-bio), in part to better align didactic teaching requirements with recommendations of the American Society for Radiation Oncology (ASTRO) Cancer Biolo-
gy/Radiation Biology Task Force for the future of research, teaching and testing in the biological sciences of radiation oncology (6).

However, the ABR outline is not a syllabus, curriculum or study guide, without which a nonradiobiologist educator might be hard pressed to know what to teach residents and in how much detail. And while the development of curricula is technically the job of the Accreditation Council for Graduate Medical Education (ACGME), there has been discussion among some senior radiation biology educators about whether a standardized curriculum in radiation and cancer biology should be developed, perhaps spearheaded by ASTRO or the RRS. This could be modeled after the detailed physics curriculum for residents developed many years ago and periodically updated by ASTRO, independent from, but that augments, the existing ABR physics outline (7).

A similar multi-step certification process applies to medical physicists, although there is not a stand-alone exam in radiation and cancer biology. Instead, clinically relevant, if limited, biology content is admixed with other parts of their written exams. Further, the Commission on Accreditation of Medical Physics Educational Programs (CAMPEP) and the ABR now require training in radiation biology (i.e., an "official" university course from a degreegranting department) for medical physics graduate students as a prerequisite for entry into an accredited medical physics residency program, which in turn is a prerequisite for certification exam eligibility. This requirement can be problematic for aspiring medical physicists in graduate programs at institutions that lack an instructor qualified to teach a radiobiology course. Again, although a general list of topics is available on the CAMPEP and ABR websites (e.g., http://www.campep.org/GraduateStandards.pdf), it is the ASTRO-developed curriculum (see above) that is used to guide most physics educators.

In the absence of a curriculum for radiation and cancer biology, what resources are available, both for trainees and their non-radiobiologist educators? One study aid, with an established track record of use by both radiation oncology and medical physics residents and their teachers (4), is the ASTRO Radiation/Cancer Biology Practice Examination and Study Guide, which is updated annually (available at http://bit.ly/1iJwUmh). This ASTRO practice exam, along with the annual American College of Radiology (ACR) In-Training exam, are used as study guides by trainees and as a source for curriculum planning and test questions by educators. Unfortunately, the pool of individuals qualified to develop these exams and accompanying study materials (all voluntary efforts) is aging and shrinking. As of eight years ago, nonradiobiologist educators comprised approximately half of the total "radiation biology" workforce (4). While no follow-up survey has been conducted since, it is reasonable to assume that this fraction will continue to increase over time. 


\section{THE DIFFICULTIES IN DEVELOPING AND EFFECTIVELY UTILIZING A CADRE OF KNOWLEDGEABLE RADIATION SCIENCES EDUCATORS}

In 2011, the ASTRO Board of Directors appointed a Cancer Biology/Radiation Biology Task Force to assess the state of basic and translational research in radiation oncology. The Task Force report ( 8 ) and summary thereof (6) contained recommendations for a timely and necessary research agenda in the radiation and cancer biology of radiation oncology. As a follow-up, ASTRO and the NCI convened a consensus workshop in 2014 to continue the discussions $(9,10)$, including a session on ways to enhance and invigorate participation and investment in radiation biology as it pertains to radiation oncology. Part of that discussion was how best to prepare current (and future) cancer and molecular biology faculty to serve as radiation biology educators and mentors for residents and others. This is of particular concern to residency program directors and to ASTRO; for the program to maintain its accreditation, the Radiation Oncology Residency Review Committee of the ACGME mandates at least one full-time radiation or cancer biologist on site to ensure that residents receive a comprehensive, didactic education in radiation and cancer biology and to serve as a research mentor.

Currently, most radiation biology educators are either basic or translational research scientists with diverse backgrounds and qualifications, ranging from senior, "classical" radiation biologists, to mid-career molecular/ cancer/tumor biologists who may or may not use radiation in their research, to, increasingly, more junior radiation oncology physician-scientists. Yet, those who come from non-radiation fields and are thrust into teaching about radiation effects find themselves needing something akin to "on-the-job training." However because of the competing pressures of conducting research, treating patients and/or obtaining grant funding in a climate of pay lines that are at or near all-time lows, many have limited interest, time and/ or motivation to undertake the training needed to become effective radiation biology educators. They (and to some extent all radiation biology educators) are often faced with limited resources for teaching or for developing new teaching materials and skills, while receiving little encouragement or incentives to teach.

Clearly, approaches need to be found to develop and use effectively the cadre of knowledgeable radiation biology educators to provide the best possible instruction for trainees in radiation oncology, medical physics and in the radiation sciences generally. Related issues that were discussed included the need for effective mentorship at all academic levels, whether "centralized" teaching (e.g., regional courses, webinars, on-line courses) could be useful or might result in too much "homogenization", and who would best teach such courses. Education-related action items proposed during the ASTRO-NCI workshop were specifically geared to radiation oncology and included the following:

- Develop clear educational "tracks" for resident physicians (e.g., clinical educators, clinical educators/researchers, physician-scientists) and elucidate the training guidelines needed for each track.

- Advocate for increased education in oncology in general, along with basic radiation effects and radiation safety at the medical student level.

- Develop two tiers of radiobiology education for residents: a basic, clinical practice-oriented knowledge base for individuals pursuing primarily clinical careers, and an approach with more in-depth coverage for the physician-scientist.

Since many in ASTRO believe that academic physicianscientists will become the de facto "radiobiologists" of the future, and will also serve as educators and mentors to residents, graduate students and post-docs, it is important to nurture their careers now. The mechanism for this is for radiation oncology departments to provide more, and longer-term, support and mentorship for early career physician-scientists, especially in the current climate of greatly reduced research funding from government agencies.

Mechanisms should be identified and available for supporting (for limited periods) established radiobiologists and physician-scientists who may be "in between" grants to ensure continuity within individual programs.

\section{DWINDLING WORKFORCE CONCERNS ARE BROAD: THE NCRP WARP (Where Are the Radiation Professionals) WORKSHOP AND INITIATIVE}

In July 2013, the National Council on Radiation Protection and Measurement (NCRP) sponsored a workshop with the goal of initiating a "Manhattan Project" to replenish the dwindling supply of radiation professionals in the U.S (5). With a broader scope than just radiation oncology, radiation biology and medical physics, the workshop included representatives of government agencies, professional societies (including RRS and ASTRO), universities, the private sector and the NCRP (5). Each speaker described his/her organization's mission or goals, and how goals were to be, or already had been, achieved, particularly with regard to education, training and development of individuals at all career levels. Unfortunately, a common theme throughout many of the presentations was the declining numbers of trained radiation professionals.

Representatives from RRS, for example, reported that the number of full society members decreased over $20 \%$ between 2003 and 2012 (5). In an attempt to reverse, or at a minimum, stabilize, the resultant shortages in biology educators and research mentors, RRS established the Scholars-in-Training (SIT) program. SIT's mission is, in part, to assist the discipline in retaining early career 
scientists who may have worked with radiation, but who do not necessarily self-identify as radiobiologists. Special SITdirected educational activities have been prominently featured at RRS meetings for approximately 15 years. While the SIT initiative has been successful in providing excellent learning and networking opportunities for graduate students, post-doctoral and early-career scientists, the continuing decline in the number of full RRS members suggests the program has had limited success with achieving that goal.

Also discussed at the WARP workshop was that in the near term (next 3-5 years), the workforce supply and demand seem fairly well balanced in the fields of radiation protection/health physics and in medical physics, with the exception of academic jobs in research and education in these fields, where very few new positions are being created. In part, the lack of academic positions in the short term may be because many baby boomers have delayed retirement or transitioned to part-time status. In the intermediate term (5-10 years), it is expected that the rate of retirement will increase, meaning that the academic job market could improve. However, there is concern that universities, when faced with further state and federal budget cuts, will not replace these retirees, which could ultimately cause academic programs to close due to lack of faculty, students or both. In the long term (10-20 years), and with fewer programs supplying fewer graduates, there is likely to be a significant shortfall in the radiation sciences workforce once the remainder of the Baby Boomer cohort moves into retirement. Unfortunately, this is projected to occur at a time when there will be a concomitant increase in demand for radiation professionals in medicine (to diagnose and treat cancers in the aforementioned baby boomer generation). A possible "nuclear energy renaissance" in the private sector could also increase demand.

To put the seriousness of this impending problem in perspective, the case of the Fukushima Daiichi nuclear power plant accident in Japan can be cited. Assessments of this incident and the U.S. response to it have shown that in the coming years, if such an incident were to occur in the U.S., there would be a shortage of sufficiently trained radiation protection professionals. $(11,12)$.

\section{OPPORTUNITIES FOR MEDICAL PHYSICISTS TO COLLABORATE WITH BIOLOGISTS AND RADIATION ONCOLOGISTS ABOUND, BUT WILL NECESSITATE ADDITIONAL TRAINING}

For the foreseeable future, there will be ample opportunity for basic and translational research focused on improving the therapeutic ratio in radiation oncology, that is, to either decrease the adverse effects of radiation therapy on normal tissues, and/or increase cure rates of patients' tumors, particularly advanced ones. Physical methods for minimizing adverse effects include more precise imaging and target identification/localization, 3D conformal irradiation, inten- sity modulation of photon beams and particle beam radiotherapy, all of which require the expertise of medical physicists. Translating basic knowledge into the clinic for decreasing the adverse effects of radiation therapy without compromising tumor control is a high priority for research (14), and will require physicists, biologists and physicians working together. Further, subsequent to the terrorist attack on the World Trade Center in 2001, a sizable investment was made by the U.S. government to develop medical countermeasures for mitigating radiation injuries in soldiers, first responders and civilians exposed to ionizing radiation (13). Much of this research falls under the purview of radiation/cancer biologists, who must work in concert with radiation physicists.

Unfortunately, today's biologists rarely receive training in radiation dosimetry, and usually use irradiators dedicated to research only, not shared with or calibrated by their physics colleagues. Likewise, there are few physicists trained in the unique characteristics of the equipment used in laboratory research and familiar with problems inherent in performing dosimetry in support of biology studies. To make matters worse, even when collaborations do occur between biologists and physicists, research progress can be stymied by the lack of a common language and common training. Physicists should be familiar with, at minimum, some of the basic principles, experimental assays and jargon of radiation/cancer biology. Biologists and physicists should both be familiar with the types of cancer that cause the most deaths due to poor tumor control after radiation therapy. Biologists also need to know about clinical radiation oncology in general. This includes, for example, knowing about the types of tumors that are readily curable with radiation therapy, but at the expense of unacceptable, adverse side effects.

Radiation dose is central to all basic and translational research in radiation biology, radiation oncology and medical physics. Precision and accuracy of dose measurements and the methods used to measure dose should be reported explicitly and in sufficient detail in publications to allow data to be interpreted, experiments reproduced and valid comparisons made (15). A careful reading of select radiobiology publications, however, suggests that measurement and reporting of radiation dosimetry and experimental setup in radiation biology research is frequently inadequate (16). This problem is only exacerbated by the increasing complexity and sophistication of radiation delivery systems and types of beams available for research, as well as the broad range of radiation biology studies that might require the irradiation of anything from macromolecules in solution, to cells in culture or to laboratory animals. If one adds to this the possibility that the "radiation biologist" in charge of the study is not a radiation biologist by training, and therefore is unfamiliar with the nuances of these types of experiments, it is perhaps not surprising that such errors of omission occur. 
These problems are not new; not only have they been discussed in the past, but detailed recommendations have been made in an attempt to standardize dosimetry reporting, particularly in biology publications $(14,16,17)$. However, to date, these recommendations have not been adopted in the U.S.

\section{SUCCESSFULLY ATTRACTING, AND RETAINING, THE NEXT GENERATION OF RADIATION RESEARCHERS}

One training approach that has been implemented recently is the "Integrated Course in Biology and Physics of Radiation Oncology" (IBPRO, http://ibpro.org/), a pilot program funded entirely by the NCI Cancer Training Branch. The IBPRO course is held once a year, with attendance limited to 50 North American participants. This advanced, weeklong course examines radiation oncology at its cutting edge, with the objectives of promoting intense discussion and discovery and encouraging future collaborations. IBPRO is targeted to biologists, physicists and clinicians (from any discipline) wishing to undertake research in radiation oncology, new researchers wishing to gain knowledge to effectively enter the field and existing clinicians, researchers and other radiation professionals wishing to refresh or advance their knowledge to state-ofthe-art. IBPRO's goals are to: reaffirm the natural liaison between radiation physics and biology, which has been a mainstay of radiation research since it began; attempt to halt the critical decline in the number of radiation biologists over the past 20 years; and reinvigorate translational and clinical radiation research. The course is designed to look forward at what needs researching, rather than reiterating what we know already, and to reaffirm and take advantage of the multidisciplinary nature of radiation biology, especially in the clinic.

Since no other course like that of the IBPRO has ever been attempted in the U.S., and there are no similar initiatives yet internationally, much of its development and implementation were from the ground up, including new support and infrastructure, the innovative curriculum and educational techniques, use of advanced conference facilities, equipment and IT support and an ongoing evaluation process that enables future activities, which change from year to year, to be planned and undertaken with maximal effect. The course includes both highly topical keynote lectures given by internationally recognized experts in biology, physics and clinical oncology (who are also expert educators adept at speaking the languages of both physics and biology), along with practical instructional activities designed to stimulate discussion and increase understanding of how unanswered questions in clinical radiation oncology and cancer biology might best be researched.

The first IBPRO, which was held in May 2014 at Wayne State University School of Medicine (Detroit, MI), was oversubscribed, but did attract the intended mix of clinicians, physicists and biologists. However, of interest is that physicists outnumbered biologists and clinicians by a ratio of 2:1:1. Attendee feedback from IBPRO 2014 (and preliminarily, from IBPRO 2015) confirm a strong interest among medical physicists for researching clinical radiobiology, perhaps spawned by recent efforts such as the Quantitative Analysis of Normal Tissue Effects in the Clinic (QUANTEC) (18) initiative and the Working Group on Stereotactic Body Radiation Therapy (WGSBRT) (19), which were spearheaded by the American Association of Physicists in Medicine (AAPM).

IBPRO was initially designed as a five-year program, with the possibility of renewed funding for a further five years. The results of the first two years' courses show that this approach is well received and likely to have a positive effect on reinvigorating radiation research, particularly from a clinical standpoint.

\section{RECOMMENDATIONS}

A number of recommendations were made at the workshop, based on the issues raised during speaker presentations and the subsequent discussion and question/ answer session with audience members. Some have been suggested at least once before but were deemed worth repeating, while others were intended to build upon or update previous recommendations. Those recommendations, at times accompanied by an explanation of the benefits of adopting or consequences of ignoring them, are listed below.

\section{Regarding General Education and Training in the Radiation Sciences:}

- Approaches are needed to develop and support educators who are broadly and deeply knowledgeable in the radiation sciences, and provide them with resources to develop novel programs and comprehensive teaching tools that are critical for the effective training of the next generation of radiation scientists. Because researchereducators play a critical but frequently overlooked and undercompensated role in the teaching mission, departments must be willing to step up efforts to retain them during temporary downturns in research funding, otherwise they risk losing experienced educators as well as researchers.

- Efforts to strengthen relationships among radiation science disciplines and societies, such as the holding of joint national meetings, should continue, as they not only help publicize the fields and attract new students, but also facilitate networking and research collaborations.

- Mechanisms must be developed to increase awareness about basic radiation effects and the existence of radiation oncology and radiation biology as viable, cutting edge medical and scientific disciplines. To 
increase the profile of radiation oncology, for example, this should start early in medical school, specifically during the first two years of training. Medical students are often unaware that radiation oncology is its own medical specialty, distinct from both radiology and medical/surgical oncology, and that it is available as an elective at many medical schools.

\section{Regarding Educational Materials and Curricula:}

- Detailed curricula should be developed covering the minimal essential knowledge in radiation and cancer biology expected of all radiation oncologists and therapeutic medical physicists. Detailed curricula would also be helpful to those educators who are not radiation biologists by training.

- ASTRO is encouraged to continue to support the yearly production of their practice exam and study guide and ideally, make it freely available to ASTRO members and non-members alike. Unfortunately, beginning with the 2015 edition, the exam has been "monetized" and is now available for free only to radiation oncology residents, with other ASTRO members and non-members charged $\$ 50$ and $\$ 75$, respectively, for access to the exam. Although this is a relatively modest charge, it could present yet another barrier for non-radiobiologist educators who are not ASTRO members to access materials for curriculum development.

Regarding Training Gaps and Opportunities in Basic and Priority Areas, and Interactions between Biologists and Physicists: ${ }^{4}$

- Biologists and physicists should collaborate on experimental study design and execution to achieve the accuracy and precision required for validation and reproducibility.

- The onus should be on both the biologist and the physicist to ensure that experimental needs are met, and this involves effective communication [this was one of the main reasons for CAMPEP's decision to require that medical physics trainees take a full course in radiobiology (16)].

- A qualified medical physicist should establish an ongoing quality assurance and dosimetric constancy program for laboratory irradiators with traceability to national or international standards.

- Journal editors and manuscript reviewers should ensure that biology authors include in their publications sufficient detail concerning the dosimetry used for their studies, including references to written standards and/or protocols used.

${ }^{4}$ Many of these recommendations were first suggested by Desrosiers et al. (16).
- A formal dosimetry intercomparison program needs to be implemented for radiobiology research, including how it would be implemented and sustained.

- Continuing education venues should be established in both the radiobiology and physics communities to foster better communication and arrive at agreed-upon standards.

- To make meaningful contributions in the priority areas of radiation mitigator/countermeasure development and radiation oncology research, education and training in the following areas is necessary:

o radiation response of healthy tissues;

$o$ intersections of radiation responses with cancer biology and drug responses;

o immunology; and

o radiation chemistry and radiation physics (including photons, protons and heavier charged particles, as well as systemic radionuclides).

- It is important to continue to attract funding for support of IBPRO and like-minded activities, and to staff these activities immediately with still-available skilled faculty who can train their future replacements, and, critically, to also encourage new researchers and teachers to enter the field.

It is the expectation and hope of all workshop participants/co-authors that the implementation of these recommendations will help raise the profile of the radiation sciences, and attract new students who will become competent researchers and educators.

\section{ACKNOWLEDGMENT}

Dr. Michael Joiner and the "Integrated course in Biology and Physics of Radiation Oncology" were supported by a grant from the National Cancer Institute's Center for Cancer Training (award no. R25CA171971).

Received: July 11, 2015; accepted: September 7, 2015; published online: October 19, 2015

\section{REFERENCES}

1. Zeman EM, Dynlacht JR, Rosenstein BS, Dewhirst MW. Toward a national consensus: Teaching radiobiology to radiation oncology residents. Int J Radiat Oncol Biol Phys 2002; 54:861-72.

2. Dynlacht, JR, Dewhirst MW, Hall EJ, Rosenstein BS, Zeman EM. Toward a consensus on radiobiology teaching to radiation oncology residents. Radiat Res 2002; 157:599-606.

3. Coleman CN, Stone HB, Alexander GA, Barcellos-Hoff MH, Bedford JS, Bristow RG, et al. Education and training for radiation scientists: Radiation Research Program and American Society of Therapeutic Radiology and Oncology Workshop, Bethesda, Maryland, May 12-14, 2003. Radiat Res 2003; 160:729-37.

4. Rosenstein BS, Held KD, Rockwell S, Williams J, Zeman EM. American Society of Radiation Oncology (ASTRO) survey of radiation biology educators in U.S. and Canadian radiation oncology residency programs. Int J Radiat Oncol Biol Phys 2009; 75:896-905.

5. National Council on Radiation Protection and Measurements. Where are the radiation professionals (WARP). Synopsis of NCRP Statement No. 12. (http://www.ncrponline.org/Publications/ Statements/WARP_Synopsis_Statement12.pdf) 
6. Wallner PE, Anscher MS, Barker CA, Bassetti M, Bristow RG, Cha YI, et al. Current status and recommendations for the future of research, teaching, and testing in the biological sciences of radiation oncology: report of the American Society for Radiation Oncology Cancer Biology/Radiation Biology Task Force, executive summary. Int J Radiat Oncol Biol Phys 2014; 88:11-7.

7. Xiao Y, Bernstein KA, Chetty IJ, Eifel P, Hughes L, Klein EE, et al. The American Society for Radiation Oncology's 2010 core physics curriculum for radiation oncology residents. Int J Radiat Oncol Biol Phys 2011; 81:1190-2.

8. Current status and recommendations for the future of research, teaching and testing in the biological sciences of radiation Oncology. Report of the American Society for Radiation Oncology Cancer Biology/Radiation Biology Task Force. ASTRO 2013. (http://bit.ly/1M6FMMy)

9. The future of radiobiology in radiation oncology. ASTRO/NCI Radiobiology Consensus Workshop, July 18, 2014, Bethesda, MD. (http://bit.ly/1EXRKsa)

10. Wallner PE, Steinberg ML, McBride WH, Hahn SM, Zietman AL. A fork in the road: Choosing the path of relevance. Int J Radiat Oncol Biol Phys 2015; 92:214-6.

11. Miller CW. The Fukushima radiological emergency and challenges identified for future public health responses. Health Phys 2012; 102:584-8.

12. Whitcomb RC, Ansari AJ, Buzzell JJ, McCurley MC, Miller CW, Smith JM, Evans DL. A public health perspective on the US response to the Fukushima radiological emergency. Health Phys 2015; 108:357-63.

13. Radiation and Nuclear Countermeasures Program. National Institute of Allergy and Infectious Diseases. (http://1.usa.gov/ 1gmaMMX)

14. Prasanna PGS, Narayanan D, Hallett K, Bernhard EJ, Ahmed MM, Evans G, et al. Radioprotectors and radiomitigators for improving radiation therapy: The small business innovation research (SBIR) gateway for accelerating clinical translation. Radiat Res 2015; 184:235-48.

15. Begley CG, Ellis LM. Drug development: Raise standards for preclinical cancer research (editorial). Nature 2012; 483:531-3.

16. Desrosiers M, DeWerd L, Deye J, Lindsay P, Murphy MK, Mitch $\mathrm{M}$, et al. The importance of dosimetry standardization in radiobiology. J Res Nat Inst Standards Technology 2013; 118:403-18.

17. Zoetelief J, Broerse JJ, Davies RW, Octave-Prignot M, Rezvani M, Vergara JC, Toni MP. Protocol for X-ray dosimetry in radiobiology. Int J Radiat Biol 2001; 77:817-35.

18. Marks LB, Ten Haken RK, Martel MK. Guest editor's introduction to QUANTEC: a users guide. Int J Radiat Oncol Biol Phys 2010; 76:S1-2.

19. Yorke E. WE-F-304-01: Overview of the working group on stereotactic body radiation therapy (WGSBRT). Med Phys 2015; 42:3685. 